Association for the Surgery of Trauma scoring system for renal injury was used to grade the kidney injuries from grade I (least severe) to grade $\mathrm{V}$ (most severe).

In total, 116 blunt renal injuries occurred, including 20 bicycle injuries (6 from dirt bikes-lightweight motorcycles), 13 all-terrain vehicle injuries (4 from rollovers), and 12 contact sport injuries. There were 45 grade I injuries, 31 grade II injuries, 23 grade III injuries, 15 grade IV injuries and 2 grade $V$ injuries. Mean renal injury grade was significantly greater when the injury was caused by a dirt bike (mean grade $2.8 \pm 1.2$ ) or an all-terrain vehicle rollover (mean grade $2.8 \pm 1.3$ ) than when it occurred during an organized contact sport such as American football or ice hockey (mean grade 1.4 $\pm 0.7 ; P=0.007$ and $P=0.02$, respectively). Only two high-grade (i.e. grade III and above) renal injuries occurred during organized contact sports, both in children not wearing protective clothing.

The authors conclude that children with a solitary kidney should be advised to avoid riding dirt bikes and all-terrain vehicles.

Original article Wu H-Y and Gaines BA (2007) Dirt bikes and all terrain vehicles: the real threat to pediatric kidneys. J Urol 178: 1672-1674

\section{Presentation and progression of atypical HUS varies with type of complement-gene mutation}

Several studies have linked mutations in the genes encoding factor $\mathrm{H}(C F H)$, factor I (CFI), and membrane cofactor protein $(M C P)$ - all regulators of complement activation - to atypical hemolytic uremic syndrome (HUS). Now, Sellier-Leclerc et al. report that the clinical characteristics of pediatric atypical HUS depend upon the form of complement dysregulation.

In a population of 46 children with atypical HUS, $52 \%$ had a mutation in at least one of the three complement-system genes examined. Patients harboring $\mathrm{CFH}$ or $\mathrm{CFI}$ mutations were markedly younger at disease onset (median ages 6 months and 2 months, respectively) than were patients with an MCP mutation (median age 4.5 years). Within 1 year of disease onset, $37 \%$ of the patients died or developed endstage renal disease (ESRD). Serum creatinine level at first flare was significantly associated with outcome at 1 year.
Patients with a $\mathrm{CFH}$ mutation had the worst prognosis, with $40 \%$ reaching ESRD at first flare and $60 \%$ progressing to this stage within 10 years. By contrast, only about $30 \%$ of patients with either an $\mathrm{MCP}$ or $\mathrm{CFI}$ mutation had reached ESRD at 10 years' follow-up. Of the 24 kidney transplantations performed in 15 patients, only 8 were successful. Interestingly, 8 of the 16 graft failures were due to vascular thrombosis rather than recurrence of atypical HUS. Identifying the mutations that underlie atypical HUS could provide valuable prognostic information and help to guide management of individual patients.

Original article Sellier-Leclerc A-L et al. (2007) Differential impact of complement mutations on clinical characteristics in atypical hemolytic uremic syndrome. J Am Soc Nephrol 18: 2392-2400

\section{Systematic review of ANCA- associated vasculitis treatments}

Bosch and colleagues searched MEDLINE for studies published in English between 1966 and 2007 that evaluated the available treatments for antineutrophil cytoplasmic antibody (ANCA)-associated vasculitis. A total of 62 observational studies or uncontrolled trials with $\geq 10$ patients, 20 prospective randomized controlled trials, and 2 meta-analyses were deemed eligible for inclusion.

The researchers found study protocols and outcomes to be heterogeneous, and agents for which most evidence is available to not be sufficiently safe or effective. Nevertheless, they made the following recommendations. For patients with isolated upper respiratory tract involvement, cotrimoxazole with or without corticosteroids can be used to induce and maintain remission. For patients with generalized, non-organ-threatening disease, methotrexate plus corticosteroids can be used to induce remission; the high risk of relapse associated with methotrexate as maintenance therapy necessitates careful monitoring during this phase of treatment. For patients with generalized, organ-threatening disease, pulse cyclophosphamide can be used with corticosteroids to induce remission, while maintenance of remission can be achieved using azathioprine plus daily prednisone. For patients with advanced renal involvement, plasma exchange is the best complement 